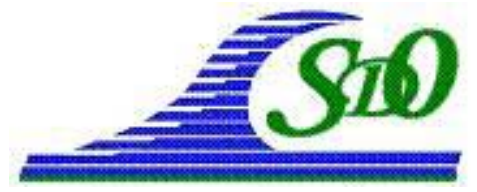

XI ${ }^{\text {èmes }}$ Journées Nationales Génie Côtier - Génie Civil

Les Sables d'Olonne, 22-25 juin 2010

DOI:10.5150/jngcgc.2010.065-S @ Editions Paralia CFL

disponible en ligne - http://www.paralia.fr - available online

\title{
Concentration en matières en suspension dans la crème de vase estuarienne par mesures optiques et acoustiques
}

\author{
Aldo SOTTOLICHIO ${ }^{1}$, David HURTHER ${ }^{2}$, \\ Philippe LAGUIONIE ${ }^{3,4}$, Caroline POULLAIN ${ }^{1,5}$
}

1. Université Bordeaux 1, EPOC, Avenue des Facultés, 33405 Talence Cedex, France. a.sottolichio@epoc.u-bordeauxl.fr

2. LEGI - UMR 5519, BP 53, 38041 Grenoble Cedex 9, France.

3. INSA Rennes, 20 av. des Buttes de Coësmes, CS 14315, 35043 Rennes, France.

4. IRSN, rue Max Pol Fouchet BP10, 50130 Cherbourg-Octeville, France.

5. EGIS BCEOM, Place des Frères Montgolfier, 78286 Guyancourt Cedex, France

\section{Résumé :}

L'objectif de ce travail est de tester la mesure de la concentration dans la crème de vase estuarienne avec deux appareils commerciaux : un vélocimètre acoustique ADV Vector Nortek@ et un sédimètre optique Argus@ $@$ ASM IV. Les résultats montrent que l'ASM s'avère très efficace pour suivre l'évolution de la lutocline, jusqu'à des concentrations d'environ $80 \mathrm{~g} \mathrm{l}^{-1}$. Le vélocimètre $\mathrm{ADV}$, pour sa part, est capable de mesurer la concentration dans une gamme qui peut atteindre $60 \mathrm{~g} \mathrm{l}^{-1} \mathrm{Ceci}$ rend potentiellement possible les mesures de flux turbulents au sein des couches de crème de vase, à condition que la mesure hydrodynamique de la turbulence soit satisfaisante. Les deux appareils sont toutefois très sensibles à la calibration du signal et notamment l'ADV, qui reste tributaire de mesures de concentration indépendantes et simultanées.

\section{Mots-clés :}

Crème de vase - Turbidité -Vélocimétrie acoustique - Turbidimètre optique - Estuaire de la Gironde - Lutocline

\begin{abstract}
:
The aim of this study is to present experiments where commercial devices were deployed to measure concentration in estuarine fluid mud: an acoustic ADV velocimeter and an optical ASM IV sedimeter. Results show that the ASM sedimeter is efficient to follow the evolution of the lutocline near the bottom, in a concentration range up to $80 \mathrm{~g} \mathrm{l}^{-1}$. The ADV velocimeter is suitable to measure concentrations up to $60 \mathrm{~g} \mathrm{l}^{-1}$. This means that is potentially possible to measure turbulent fluxes in fluid mud, provided that turbulence measurement is accurate. The two devices are very sensitive to their calibration and the ADV is strongly dependent on simultaneous measurements of concentration.
\end{abstract}




\section{Introduction}

Le dépôt de crème de vase sur le fond des chenaux et des ports, ainsi que son évolution dans des conditions naturelles ont été observés depuis des années (KIRBY, 1988). Cependant, les processus physiques qui contrôlent sa formation ont été étudiés le plus souvent en laboratoire (WOLANSKI et al., 1989; GRATIOT et al., 2005) ou par modélisation numérique (LE HIR et al., 2001 ; WINTERWERP et al., 2001 ; par exemple). L'analyse de ces processus dans le milieu naturel et la validation des modèles nécessitent la mesure simultanée du champ turbulent et des concentrations en sédiments en suspension dans les conditions de la crème de vase in situ. Cependant, la très forte concentration en sédiments est une limite importante à ce type de mesure car les particules absorbent une grande partie des signaux acoustiques et optiques, de telle sorte que les instruments de mesures classiques se trouvent a priori limités. Pour ces mêmes raisons, la performance réelle de ces appareils n'a pas encore été suffisamment testée en milieu naturel. Le développement récent d'instrumentation physique plus performante et mieux adaptée aux conditions naturelles rend progressivement possible la mesure conjointe des vitesses et des concentrations. En particulier, les vélocimètres acoustiques ADV sont potentiellement les plus pertinents, puisqu'en plus de la mesure de vitesse, ils peuvent fournir une mesure de concentration par l'étalonnage de leur signal acoustique (VERNEY et al., 2007 ; HA et al., 2009). L'objectif de ce travail est de présenter les premiers résultats de travaux menés dans l'estuaire de la Gironde, au cours de laquelle ont été testés simultanément un vélocimètre acoustique et un turbidimètre laser optique dans un milieu naturel fortement concentré (gammes au-delà de la dizaine de $\mathrm{g} \mathrm{l}^{-1}$ ).

\section{Matériel et méthodes}

Ce travail repose sur l'utilisation conjointe et complémentaire de deux instruments : un sédimètre Argus@ $@$ ASM-IV, et un vélocimètre ADV Vector Nortek@. Pour ce dernier, nous présentons les résultats correspondant à la mesure de la concentration en sédiments uniquement. Chacun des appareils a fait l'objet d'une calibration préalable et indépendante en laboratoire, puis d'un déploiement simultané dans le milieu naturel.

\subsection{Le sédimètre ASM-IV}

Le sédimètre submersible ASM-IV est constitué d'une tige rigide de 1,5 $\mathrm{m}$ de long et 3 $\mathrm{cm}$ de diamètre, le long de laquelle sont disposés 144 capteurs laser infrarouges $(850 \mathrm{~nm}$ ), espacés régulièrement de $1 \mathrm{~cm}$ (figure 1a). La perche est surmontée d'une tête de $60 \mathrm{~mm}$ de diamètre, contenant un inclinomètre, des capteurs de température et de pression, batterie et mémoire de stockage. Chacun des capteurs mesure à la fréquence minimum de $1 \mathrm{~Hz}$ une "réflectivité" qui correspond à la lumière rétrodiffusée par les particules en suspension et qui est exprimée en "counts" selon une échelle entre 0 et 4095 (LAGUIONIE, 2006). La calibration en laboratoire a consisté à associer les valeurs de réflectivité de chacun des capteurs à des valeurs de concentration en matière 
en suspension. Au laboratoire (INSA de Rennes, figure 1a), le sédimètre a été positionné verticalement dans une colonne de plexiglas de $2 \mathrm{~m}$ de long et $30 \mathrm{~cm}$ de diamètre dans laquelle a été versé un mélange d'eau et de vase naturelle prélevée dans l'estuaire de la Gironde. La vase a été préalablement lyophilisée de manière à obtenir une poudre pouvant être versée dans le mélange de manière contrôlée. Pendant la durée de la calibration, un brassage a été assuré afin de maximiser l'homogénéité verticale de la concentration dans la colonne. Au total, 25 concentrations différentes ont été obtenues entre 0,4 et $77 \mathrm{~g} \mathrm{l}^{-1}$. Pour chaque valeur de concentration, le sédimètre a enregistré la réflectivité des 144 capteurs pendant 5 minutes à la fréquence de $1 \mathrm{~Hz}$.

\subsection{Le vélocimètre Vector ADV}

Le modèle de vélocimètre utilisé est le Vector de chez Nortek. Il possède un émetteur acoustique (signal à $6 \mathrm{MHz}$ ) et trois récepteurs permettant de mesurer les trois composantes de la vitesse dans le volume insonifié situé à $15 \mathrm{~cm}$ de distance de l'émetteur. La calibration en concentration exploite l'intensité acoustique rétrodiffusée, reçue en "counts" (proportionnels à des $\mathrm{dB}$ ). Pour réaliser la calibration en laboratoire (EPOC Talence), nous avons utilisé un container en plastique dans lequel la sonde ADV a été immergée verticalement (figures $1 \mathrm{~b}$ et 1c). Le container est rempli initialement d'une vase fluide de concentration d'environ $500 \mathrm{~g} \mathrm{l}^{-1}$; puis progressivement, le mélange est dilué par vidanges et ajouts successifs d'eau douce. L'opération est répétée jusqu'à l'obtention d'une eau "claire" dans le container. Pour chaque palier de concentration, l'ADV mesure en continu pendan 11 min à la fréquence de $16 \mathrm{~Hz}$.

\subsection{Déploiement in situ}

Des expérimentations ont été menées dans le cadre des campagnes océanographiques ADV et REAGIR, en 2004 et 2005 (SOTTOLICHIO et al., 2007). L'estuaire de la Gironde est un estuaire macrotidal connu pour des concentrations dans le bouchon vaseux très supérieures à $1 \mathrm{~g} \mathrm{l}^{-1}$. La crème de vase se dépose surtout dans le chenal de navigation, et principalement en périodes de marée décroissante, pouvant former des couches de plus de 1 mètre d'épaisseur en quelques heures, avec des concentrations de 100 à $300 \mathrm{~g} \mathrm{l}^{-1}$ (LE HIR et al., 2001). Les résultats présentés ici ont été obtenus lors de la campagne REAGIR 4 du 5 au 12 novembre 2005. La mise en place des instruments in situ a consisté à déployer une structure benthique autonome sur laquelle ont été fixés l'ADV et le sédimètre ASM. Le Vector était en position verticale à $10 \mathrm{~cm}$ de distance du sédimètre ASM, et le volume de mesure à $1,15 \mathrm{~m}$ de hauteur au-dessus de la base de la structure (figure 1d). La structure a été immergée à plusieurs reprises pour des cycles de 12 à 24 h, dans une zone située entre le bord et l'axe du chenal de navigation, le long de la rive gauche de l'estuaire, à $45 \mathrm{~km}$ en amont de l'embouchure. 

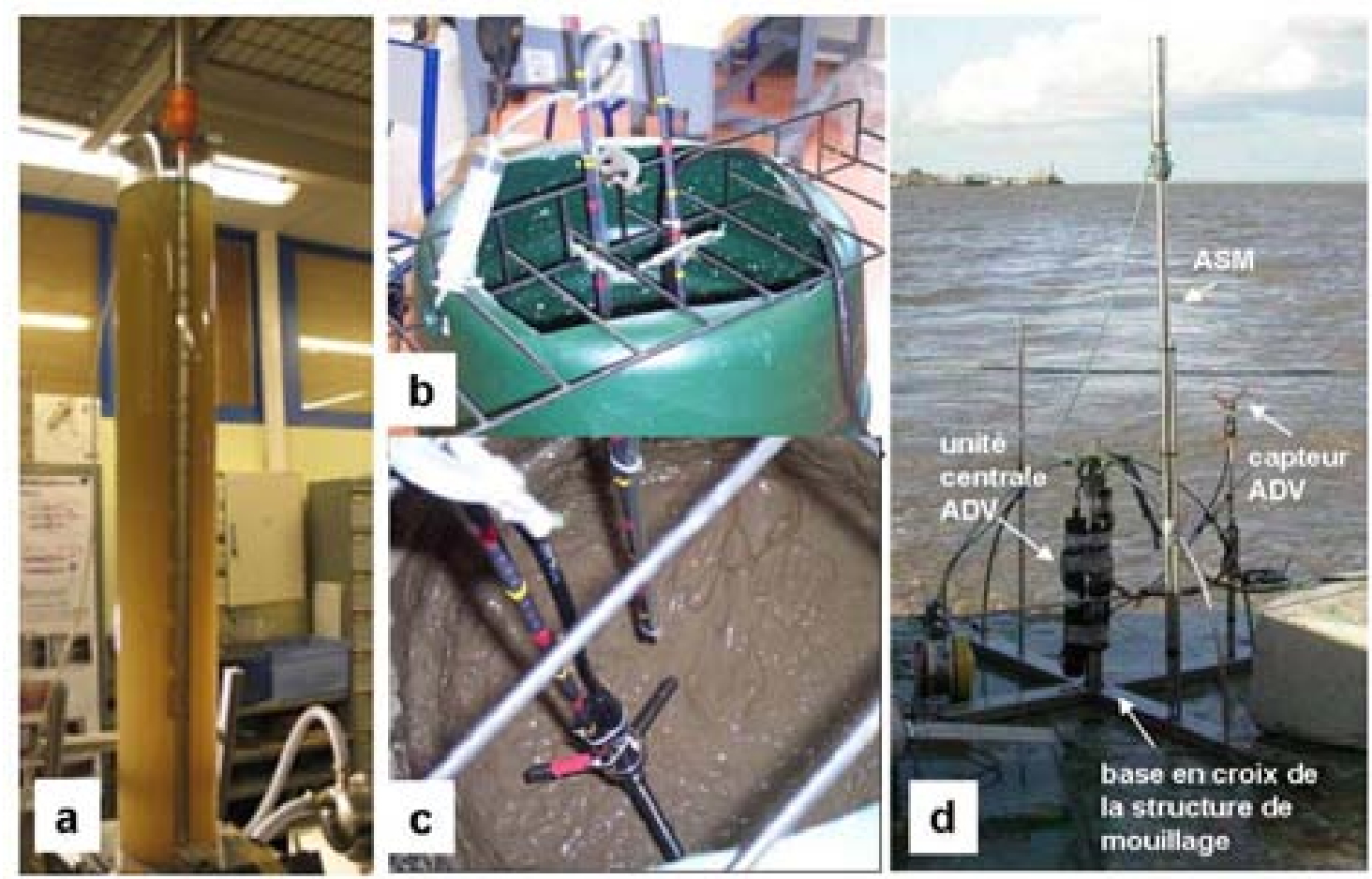

Figure 1. a) sédimètre ASM IV dans la colonne de calibration ; b) et c) ADV Vector dans le container de calibration; d) structure de mouillage à bord du N/O Côte de la Manche, avant sa mise à l'eau dans l'estuaire de la Gironde.

\section{Résultats}

\subsection{Calibration des sondes en laboratoire}

La figure 2a montre la courbe expérimentale reliant la réflectivité moyenne des 144 capteurs de l'ASM IV à la concentration moyenne mesurée dans la colonne de calibration. La relation est monotone dans toute la gamme de concentrations étudiées, avec une bonne dynamique de variation des réflectivités dans les basses concentrations. Au-delà 30-40 $\mathrm{g} \mathrm{l}^{-1}$, la réflectivité moyenne varie peu avec la concentration. Ceci indique une forte sensibilité des réflectivités aux grandes concentrations, qui atteignent un maximum de $77 \mathrm{~g} \mathrm{l}^{-1}$ au cours de l'étalonnage. Sur le graphique sont reportées également les réponses individuelles de 8 capteurs pris le long de la tige. On peut remarquer que pour une même concentration, l'écart en réflectivité peut être important entre deux capteurs extrêmes (1 et 142) ; cet écart est plus important dans les fortes valeurs de réflectivité et de concentration. Ceci semble être la conséquence d'une homogénéisation verticale insuffisante du mélange dans la colonne de calibration au cours des expériences. Par ailleurs, il est important de souligner que la réponse continue de l'ASM suggère que la rétrodiffusion est dominante malgré les fortes concentrations, et que le domaine de l'absorption ne semble pas atteint lors de l'expérience. 


\section{XI $I^{\text {èmes }}$ Journées Nationales Génie Côtier - Génie Civil}

Les Sables d'Olonne, 22-25 juin 2010

En ce qui concerne le vélocimètre $\mathrm{ADV}$, la figure $2 \mathrm{~b}$ illustre la courbe de calibration obtenue en laboratoire. Il y une bonne correspondance entre l'intensité acoustique reçue par la sonde et la concentration dans la gamme 1-60 $\mathrm{g} \mathrm{l}^{-1}$. Les variations de l'intensité montrent clairement un domaine de rétrodiffusion (pour des concentrations inférieures à $5,5 \mathrm{~g} \mathrm{l}^{-1}$ environ) où l'intensité augmente avec la concentration, et un domaine d'absorption, au-delà de $5,5 \mathrm{~g} \mathrm{l}^{-1}$, où l'intensité diminue (au-delà de $30 \mathrm{~g} \mathrm{l}^{-1}$ l'échelle ne $^{\prime}$ permet plus de voir la diminution). Par cette méthode de calibration, nous obtenons deux équations de régression entre l'intensité acoustique de l'ADV et la concentration jusqu'à $60 \mathrm{~g} \mathrm{l}^{-1}$. Toutefois, l'application de l'une ou de l'autre nécessite de savoir à tout moment si, à intensité acoustique égale, la mesure est effectuée dans un milieu diffusant ou absorbant.
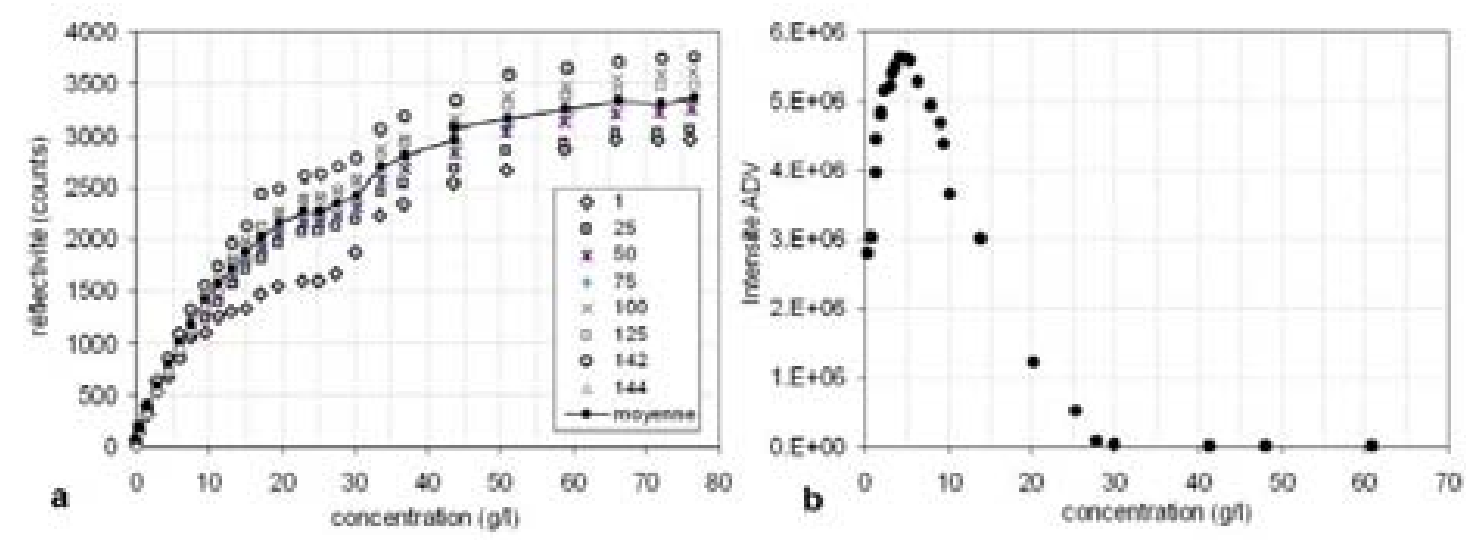

Figure 2. Etalonnage des capteurs en laboratoire. a) Sédimètre ASM IV. Huit capteurs sur 144 sont représentés (numérotés selon leur position sur la tige); la courbe relie les points de la réflectivité moyenne des 144 capteurs pour une concentration moyenne dans la colonne. b) Vélocimètre ADV Vector.

\subsection{Mesure de la concentration in situ par le sédimètre ASM}

La période de mesure correspond à des marées de mortes-eaux, favorables au dépôt de crème de vase dans l'estuaire. Plusieurs immersions de la structure instrumentée ont été effectuées, pour des gammes de concentration variées. Sur la figure 3 est présenté un exemple d'évolution de la concentration mesurée par le sédimètre ASM-IV et moyennée sur 1 min lors de deux cycles de marée consécutifs, au-dessus d'un fond vaseux. La courbe d'étalonnage présentée en figure 2 a a été extrapolée jusqu'aux plus fortes valeurs de réflectivité mesurées sur le terrain (supérieures à celle du laboratoire), de telle sorte que les concentrations calculées dépassent $80 \mathrm{~g} \mathrm{l}^{-1}$. Le premier cycle démarre à la fin de la basse mer, comme indiqué par l'enregistrement de la pression (figure 3a). Sur la figure $3 \mathrm{~b}$ on peut suivre l'évolution de la concentration, qui présente une forte dynamique, avec des phases d'augmentation et de chute rapide. Les plus fortes concentrations dépassent $120 \mathrm{~g} \mathrm{l}^{-1}$ dans les 40 premiers $\mathrm{cm}$ au-dessus du fond, ce qui 
indique bien la présence de crème de vase. Lors des phases de chute de concentration, la crème de vase la plus concentrée disparaît et la concentration peut descendre jusqu'à $1 \mathrm{~g} \mathrm{l}^{-1}$ dans la colonne d'eau. Ces deux phases très contrastées sont illustrées sur la figure $3 \mathrm{c}:$ à $8 \mathrm{~h}$ on voit l'existence d'une lutocline à $70 \mathrm{~cm}$ au-dessus du fond, séparant une couche d'eau turbide $\left(\mathrm{C}<2 \mathrm{~g} \mathrm{l}^{-1}\right)$ d'une couche de crème de vase concentrée (C>100 $\mathrm{g} \mathrm{l}^{-1}$ ). A 12h, la lutocline a disparu, la concentration est plus faible mais plus homogène, entre 5 et $20 \mathrm{~g} \mathrm{l}^{-1}$, suggérant la présence d'une crème de vase plus fluide.
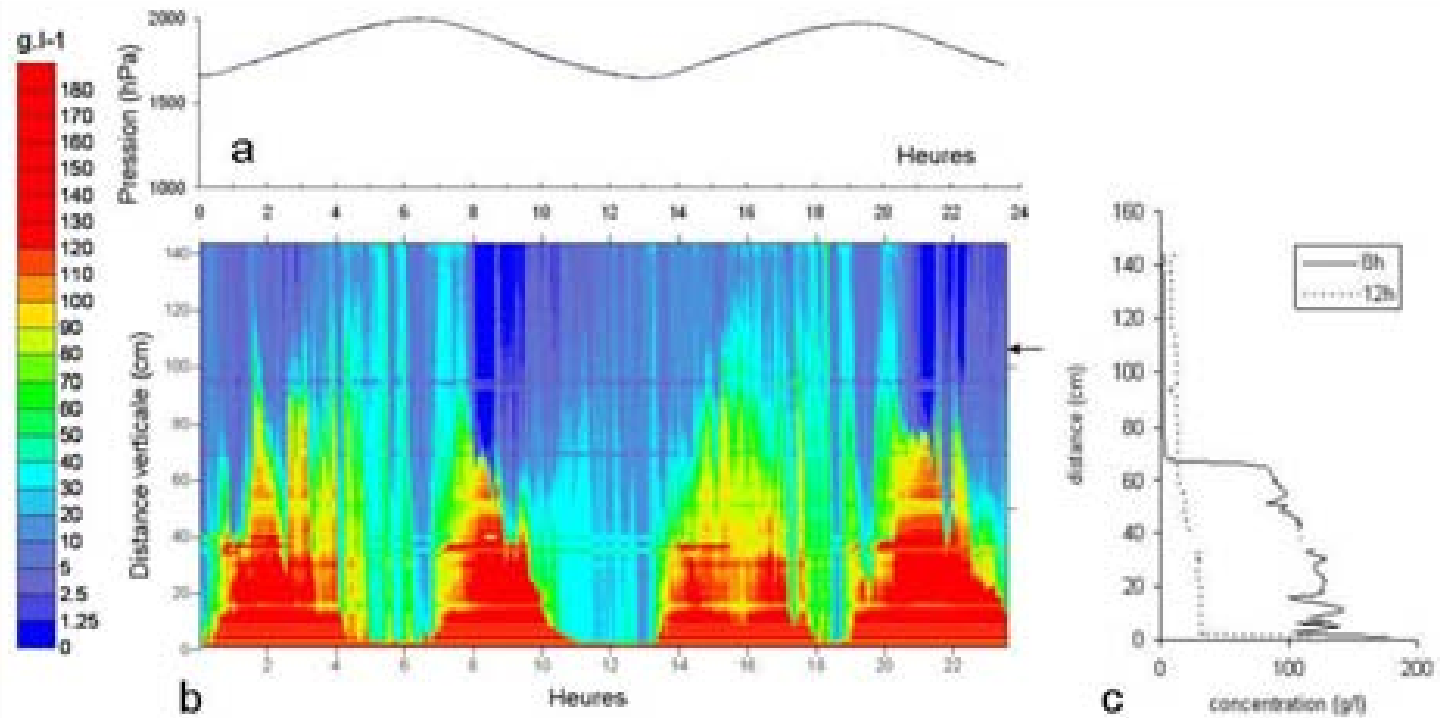

Figure 3. Enregistrements du sédimètre ASM IV dans l'estuaire de la Gironde, le 10/11/2005. a) marée ; b) concentration près du fond (la flèche à droite indique la hauteur de l'ADV) ; c) profils verticaux instantanés de concentration à $8 \mathrm{~h}$ et à $12 \mathrm{~h}$

\subsection{Concentration in situ mesurée par l'ADV}

La concentration mesurée par l'ADV a été comparée à la concentration du capteur de l'ASM situé à la même hauteur $(115 \mathrm{~cm})$. En même temps, les concentrations ASM ont servi de référence pour déterminer si le milieu était en régime de diffusion ou d'absorption, et ainsi pour choisir à chaque enregistrement la bonne équation d'inversion des intensités de l'ADV Vector. La figure 4 montre que la dynamique de la concentration est bien restituée par l'ADV : les oscillations à haute fréquence entre les heures 2 et 6 , le pic de concentration suivi d'une forte chute entre les heures 7 et 8 , puis l'augmentation progressive et irrégulière entre les heures 10 et 19 sont bien corrélés avec la mesure du sédimètre ASM. Toutefois des différences importantes peuvent exister à certains moments dans les valeurs de concentrations des deux appareils. L'inversion ADV semble surestimer la concentration lors de certains événements (heures 1 à 2 , heure 11, heure 15 , et de 20 à 22), et elle la sous-estime à d'autres (heures 4 à 6 , et 16 à 19). 


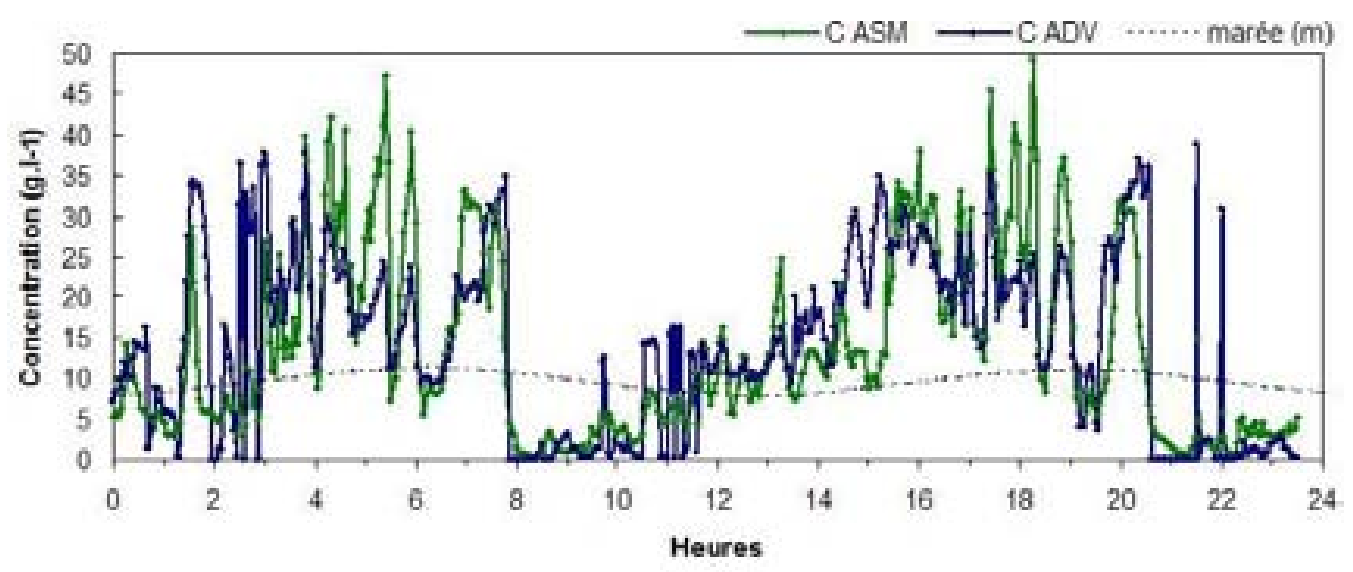

Figure 4. Comparaison des concentrations mesurées par le sédimètre ASM (C-ASM) et par le Vector $(C-A D V)$ à $115 \mathrm{~cm}$ au-dessus du fond pendant 2 cycles de marée. Les mesures à $32 \mathrm{~Hz}$ ont été moyennées sur 3 min pour filtrer les fluctuations turbulentes.

\section{Discussion et conclusion}

Il y a globalement une bonne correspondance entre les concentrations obtenues par les méthodes optiques et acoustiques, même si l'écart de concentration peut parfois atteindre $100 \%$ (heures 5 et 15 par exemple, figure 4). Pour des situations mesurées à des concentrations plus basses $\left(<20 \mathrm{~g} \mathrm{l}^{-1}\right.$, non présentées ici), la correspondance s'améliore sensiblement. Il faut rappeler que les calibrations en laboratoire ont été effectuées de manière indépendante, avec des échantillons de vase différents. Ceci peut expliquer une réponse différente des deux capteurs pour une même concentration, mais indique aussi une bonne performance des appareils sur le terrain, au vu de la cohérence des mesures. Par ailleurs, la réponse de l'ADV est très sensible dans les fortes concentrations $\left(>30 \mathrm{~g} \mathrm{l}^{-1}\right)$. Une faible variation d'intensité acoustique se traduit par une forte variation de concentration, pouvant entraîner des erreurs importantes, que l'on retrouvera moins dans des gammes de concentration inférieure.

En ce qui concerne le sédimètre ASM IV, sa performance est prometteuse. Toutefois, la pertinence de l'extrapolation de notre étalonnage au-delà de $77 \mathrm{~g} \mathrm{l}^{-1}$ est à vérifier, notamment en analysant la transition rétrodiffusion/absorption, encore peu documentée pour cet instrument. L'étalonnage avec la tige en position horizontale devrait aussi aider à optimiser l'homogénéité des concentrations et à affiner la calibration de son signal.

Enfin, la réponse de l'ADV dans une large gamme de concentration semble ne dépendre que du matériel en suspension. En effet, pour un étalonnage obtenu avec un ADV différent (HA et al., 2009) les réponses sont comparables, et le passage rétrodiffusion/absorption se fait dans des gammes de concentration similaires. Il faut souligner que pour une bonne inversion des mesures ADV sur le terrain, il est fondamental d'avoir une information indépendante sur la concentration dans le milieu, afin de déterminer si la régression à utiliser est celle obtenue en rétrodiffusion ou en absorption. Une alternative pour lever toute ambigüité serait de pouvoir utiliser 
plusieurs vélocimètres superposés (2 ou 3), à l'appui de l'hypothèse d'un profil de concentration croissante vers le fond.

Les mesures de turbulence par l'ADV, non présentées ici, doivent être analysées. En effet, la capacité du vélocimètre à mesurer des flux turbulents dans la crème de vase dépendra de la qualité de la mesure hydrodynamique. Le couplage avec le sédimètre ASM s'avère potentiellement intéressant pour suivre le flux turbulent près du fond : la sonde acoustique fournit une mesure fine et ponctuelle, la tige laser optique fournit le champ vertical de concentration avec notamment la position de la lutocline.

\section{Remerciements}

Les auteurs remercient A. Crave pour sa participation aux campagnes, A. Héquette, J-C. Brun Cottan et $\mathrm{S}$. Abadie pour la mise à disposition de leurs vélocimètres $\mathrm{ADV}$.

\section{Références bibliographiques}

GRATIOT N., MICHALLET H., MORY M. (2005). On the determination of the settling flux of cohesive sediments in a turbulent fluid. Journal of Geophysical Research, vol. 110, C06004. doi:10.1029/2004JC002732

HA H.K., HSU W.-Y., MAA J.P.-Y., SHAO Y.Y., HOLLAND C.W. (2009). Using ADV backscatter strength for measuring suspended cohesive sediment concentration. Cont. Shelf Res., 29, pp 1310-1316. doi:10.1016/j.csr.2009.03.001

KIRBY R. (1988) High concentration suspension (fluid mud) layers in estuaries. In: Physical Processes in Estuaries, L.J. Dronkers, W. Van Leussen (Eds.), SpringerVerlag, pp 463-487.

LAGUIONIE P. (2006). Mesures in situ et modélisation du transport des sédiments en rivière. Application au bassin versant de la Vilaine. Thèse Univ. Rennes 1, 3497, 331 p. LE HIR P., BASSOULLET P., JESTIN H. (2001). Application of the continuous modelling concept to simulate high-concentration suspended sediment in a macrotidal estuary. In : W.H. McAnally, A.J. Mehta (eds.), Coastal and Estuarine Fine Sediments Processes, Elsevier, Amsterdam, pp 229-247.

SOTTOLICHIO A., HURTHER D., GRATIOT N., BRETEL P. (2007). Benthic processes in a highly turbid estuary through acoustic velocity measurements. 9th INTERCOH Conference, September 25-28, 2007, Brest-France, 2 p.

VERNEY R., DELOFFRE J., BRUN-COTTAN J.-C., LAFITE R. (2007). The effect of wave-induced turbulence on intertidal mudflats: Impact of boat traffic and wind. Cont. Shelf Res., 27, pp 594-612. doi:10.1016/j.csr.2006.10.005

WINTERWERP J.C., UITTENBOGAARD R.E., DE KOK J.M. (2001). Rapid siltation from saturated mud suspensions. In: Coastal and Estuarine Fine Sediments Processes, W.H. McAnally, A.J. Mehta (eds.), Elsevier, Amsterdam, pp 125-146.

WOLANSKI E., ASAEDA T., IMBERGER J. (1989). Mixing across a lutocline. Limnol. Oceanogr., 34(5), pp 931-938. 